\title{
Oral Resveratrol supplementation attenuates exercise- induced Interleukin- 6 but not Oxidative Stress after a high intensity cycling challenge in adults
}

\author{
Jung-Piao Tsao"\#, Chia-Chen Liu", Hsueh-Fang Wang2\#, Jeffrey R. Bernard ${ }^{1}$, Chun-Ching Huang4 and \\ I-Shiung Cheng $1^{\bowtie}$ \\ 1. Department of Physical Education, National Taichung University of Education, Taichung City, Taiwan. \\ 2. Department of Nutrition, Institute of Biomedical Nutrition, Hungkuang University, Taichung City, Taiwan. \\ 3. Department of Kinesiology, California State University, Stanislaus, Turlock, CA, USA. \\ 4. Department of Exercise and Health Science, National Taipei University of Nursing and Health Science, Taipei City, Taiwan. \\ \#These authors contributed equally to this work.
}

$\square$ Corresponding author: I-Shiung Cheng, Address: No. 140, Minsheng Rd., West Dist., Taichung City 40306, Taiwan. E-mail: ischeng1965@mail.ntcu.edu.tw; Tel.: 886-4-22183459.

(c) The author(s). This is an open access article distributed under the terms of the Creative Commons Attribution License (https://creativecommons.org/licenses/by/4.0/). See http://ivyspring.com/terms for full terms and conditions.

Received: 2020.11.08; Accepted: 2021.02.19; Published: 2021.03.18

\begin{abstract}
Previous studies demonstrated that resveratrol (RES) is able to enhance antioxidant, anti-inflammatory and insulin actions in humans. It is unclear whether RES can be used as ergogenic aids to enhance high-intensity cycling exercise performance and attenuate the high-intensity exercise-induced oxidative stress and inflammation. This study investigated the effect of RES supplementation on oxidative stress, inflammation, exercise-induced fatigue, and endurance performance. Eight male athletes participated in this single-blind crossover designed study and randomly instructed to receive four days of either oral RES (480 mg per day, totally $1920 \mathrm{mg}$ ) or placebo supplementation. The cycling exercise challenge at $80 \%$ maximal oxygen consumption with $60 \mathrm{rpm}$ was performed following 4 days of either RES or placebo supplementation. The total cycling performance time was recorded. In addition, blood samples were obtained to analyze the changes in blood glucose, plasma non-esterified fatty acid, serum lactate dehydrogenase, creatine kinase, uric acid, total antioxidant capacity, malondialdehyde, tumor necrosis factor- $\alpha$, and interleukin-6. The exhausting time of cycling exercise challenge was not significantly increased in RES compared to that in placebo. However, IL-6 response was significantly decreased during exercise challenge in RES trial, and there were no differences in blood biomarkers, fatigue factors, and antioxidative response. Oral RES supplementation can attenuate exercise-induced IL- 6 response but not fatigue and oxidative stress, inflammation response. However, we infer that 4-day oral RES supplementation has no ergogenic property on enhancing the high-intensity cycling exercise performance.
\end{abstract}

Key words: antioxidant phytochemicals; ergogenic property; cycling exercise; fatigue

\section{Introduction}

Resveratrol (trans-3, 4', 5-trihydroxystilbene, RES) is a naturally occurring polyphenol [1] with antioxidant [2], anti-inflammatory [3] properties. Resveratrol reported to increase insulin sensitivity [4] and accelerates fat oxidation [5]. However, the effect of RES supplementation on oxidative stress, inflammation and energy metabolism remains unclear in humans following exercise challenge. High- intensity exercise trigger reactive oxygen species (ROS) production and cause oxidative stress and inflammation in humans, which eventually aggravate fatigue and decrease exercise performance [6, 7]. Pro-inflammatory cytokines, including interleukin-6 (IL-6) and tumor necrosis factor-alpha (TNF-a) were reported to increase in rat skeletal muscle after a 90-min downhill running [8]. Administration of RES 
(50 mg/ kg) with self-nanoemulsifying drug delivery system exerted anti-fatigue effect in exhaustive swimming rats [9]. Another study demonstrated that rats with low doses of RES for 21 consecutive days significantly enhanced the swimming endurance performance. The supplementary effect of RES was notable regardless of its dosage $(25,50$ or $125 \mathrm{mg} / \mathrm{kg})$, and a dose-dependent effect was observed due to its antioxidant and anti-inflammatory properties [10].

Human trials revealed that RES supplementation improved maximum oxygen uptake ( $\left.\dot{\mathrm{V}} \mathrm{O}_{2 \max }\right)$, Wingate anaerobic test peak power, peroxisome proliferator- activated receptor gamma coactivator 1-a (PGC-1a), Sirtuin 1 (SIRT1) level and superoxide dismutase 2 (SOD2) activity after 4-week high-intensity interval training. However, the synergistic effects of RES supplementation and exercise training were not observed according to this human study by Scribbans [11]. Another human study demonstrated that shorter-term RES supplementation (4-day) could not enhance glycogen replenishment and mitochondria biosynthesis in exercised skeletal muscle following a $70 \% \dot{\mathrm{V}} \mathrm{O}_{2 \max }$ cycling exercise challenge for 60 minutes [12]. The physiological impact of high-intensity exercise may have masked the RES effect on augmenting the performance in these two human studies.

Interleukin-6, a pro-inflammatory cytokine is an important indicator of glycogen metabolism during exercise period [13], which concomitants a progressive decline of muscle glycogen content after a prolong exercise in humans [14]. High-intensity cycling exercise produced an abruptly systemic IL-6 response involving a significant muscle mass in the contractile activity. Of course, oxidative stress induced after a high-intensity exercise challenge can increase pro-inflammatory cytokines, such as IL-6 and tumor necrosis factor- $\alpha$ (TNF- $\alpha$ ) reactions $[15,16]$. Therefore, attenuation of pro-inflammatory response may depend on the rapid elimination of oxidative stress during athletic performance. In vitro or animal studies showed that RES significantly reduces oxidative stress and positively affects anti-inflammatory reactions [17]. In particular, animal studies have shown that RES significantly alleviates exercise-induced fatigue and suppressed oxidative stress $[10,18,19]$. It has been further documented that the oral bioavailability of RES was increased and the swimming time to exhaustion was also significantly increased after RES using self-micro emulsifying drug delivery system [9].

4-day oral RES supplementation (480 mg per day, totally $1920 \mathrm{mg}$ ) was conducted in the present human study. The dosage of resveratrol used and duration of supplementation period employed from the calculating on the efficient rodent dosage and duration [9]. The relevant human study of similar resveratrol products was conducted by Huang et al. on muscle cell mitochondria biosynthesis with 4 days of oral supplementation during the post-exercise recovery period [12]. Therefore, the purpose of our human study was to clarify the short-term ergogenic property of resveratrol on attenuating oxidative stress, pro-inflammation, as to eliminate exerciseinduced fatigue under the cycling exercise at $80 \%$ $\dot{\mathrm{V}} \mathrm{O}_{2 \max }$ with $60 \mathrm{rpm}$.

\section{Materials and Methods}

\section{Participants}

Eight male participants, physically active students, with an average age of $19.2 \pm 0.5$ years and an average body mass index (BMI) of $23.3 \pm 1.8$ and an average $\dot{\mathrm{V}} \mathrm{O}_{2 \max }$ of $51.4 \pm 1.7 \mathrm{~mL} / \mathrm{kg} / \mathrm{min}$ were recruited. All participants attended the experiment design explanation and provided informed consent before beginning the experimental process. During the experimental period, the participants were required to avoid consuming pungent beverages (e.g., coffee, tea, cola, and chocolate), drugs and nutritional products which caused oxygen stress, inflammation response or changes in metabolism status will be suggested to refrain from consuming by subjects during experimental period. The investigations were carried out following the rules of the Declaration of Helsinki of 1975 and this study was approved on August 1st, 2017 by the Institutional Review Board in University of Taipei, Taipei, Taiwan (approval license IRB-2016-053). All participants joined the experiment voluntarily, for 2 months prior to the formal experiment and avoided taking supplements with anti-inflammatory agents. All subjects were fully informed of the risks and discomfort associated with the study, and all provided voluntary written consent before participation.

\section{Experiment design and procedure}

All subjects performed this single-blind crossover study design with a 7-day washout period between each trial. The participants' maximal oxygen consumption $\left(\dot{\mathrm{V}}_{2 \max }\right)$ test was measured 7 days before each single bout of exercise challenge. Starting 3 days before each exercise challenge, the participants were reminded to avoid intense exercise performing, coffee consumption, and smoking. The participants consumed 300-calorie breakfasts each day $(60 \%$ carbohydrates, $25 \%$ fat, and $15 \%$ protein), and received 480-mg RES supplements or placeboes; on the fourth day, 60 min after RES supplementation, the participants began to perform a cycling challenge 
(Monark Exercise, Varberg, Sweden). A 5-min warmup exercise was conducted with a resistance of $50 \mathrm{~W}$. Then, a 60-rpm cycling endurance test was performed at $80 \%$ of $\dot{\mathrm{V}} \mathrm{O}_{2 \max }$, and the time of challenge completion was recorded. The participants were asked to stop the workout and record the time after meeting two of three conditions: (1) unable to maintain the default exercise intensity with heart rate at maximum (220 - age); (2) oxygen uptake no longer increasing and respiratory exchange ratio (RER) $>1.1$; (3) rated perceived exertion (RPE) level $\geq 18$. Before, during, and after each workout, blood samples were collected to measure the concentrations of glucose, non-esterified fatty acids (NEFA), lactate dehydrogenase (LDH), creatine kinase (CK), uric acid (UA), total antioxidant capacity (TAC), malondialdehyde (MDA), TNF- $a$, and IL-6.

\section{Maximal oxygen consumption test}

The participants wore masks to complete the $\dot{\mathrm{V}} \mathrm{O}_{2 \max }$ measurement on cycle ergometers while using gas analyzers. The ergometers were required to maintain a rotating speed of $60 \mathrm{rpm}$. The load was initially set at $0.5 \mathrm{~kg}$ for $4 \mathrm{~min}$, and then increased by $0.5 \mathrm{~kg}$ at 2-min intervals until the participants were exhausted. The $\dot{\mathrm{VO}_{2 \max }}$ was required to satisfy the following criteria: (1) RER > 1.10; (2) $\mathrm{V}_{2}$ max variance $<2 \mathrm{~mL} / \mathrm{kg} / \mathrm{min}$; and (3) heart rate target reaches maximum heart rate at (220 - age) [20].

\section{RES and placebo supplementation}

During the 4-day RES supplementation, the participants consumed breakfast in the laboratory at 8:00 AM each day. After breakfast, the participants received oral RES supplementation comprising three $160-\mathrm{mg}$ capsules (a total of $480 \mathrm{mg}$ ) or three placebo capsules. The RES capsule contained a mixture of resveratrol with maltodextrin and lecithin (Taiwan Jellyfig Biotechnology Corporation, Kaohsiung, Taiwan), the placebo capsules with starch. The RES capsules were nanocapsules created using selfmicroemulsifying drug delivery system technology, containing $160 \mathrm{mg}$ of RES extracted from peanuts. These RES capsules had higher absorption rate than that of commercially available RES [9].

\section{Blood biochemical assessments}

Vacuum blood collection tubes containing heparin anticoagulant were employed to collect blood samples from the radial veins of the participants. The samples were centrifuged at $4{ }^{\circ} \mathrm{C}$ and $1,000 \mathrm{~g}$ for 10 min. Plasma from the supernatant was then collected and preserved at $-80^{\circ} \mathrm{C}$ in a freezer.

Blood glucose concentration was determined by an automated glucose analyzer (YSI Life Sciences). NEFA, LDH, CK, and UA concentrations were measured using the commercialized assay kit with an automatic photometric analyzer (Hitachi 7020, Japan), NEFA reagents (Randox, Ransel, UK), and LDH, CK, and UA reagents (Kanto Chemical, Kanagawa, Japan). The results were converted to concentration units. TAC and MDA indices, which are relevant to antioxidant capacity and oxidative stress respectively, were measured using a commercial TAC and MDA reagent (Cayman Chemical Company, ANN Arbor, MI, USA). An enzyme immunoassay (Tecan GENios, A-5082, Austria) was applied to measure the absorbance at $570 \mathrm{~nm}$ and the TAC and MDA

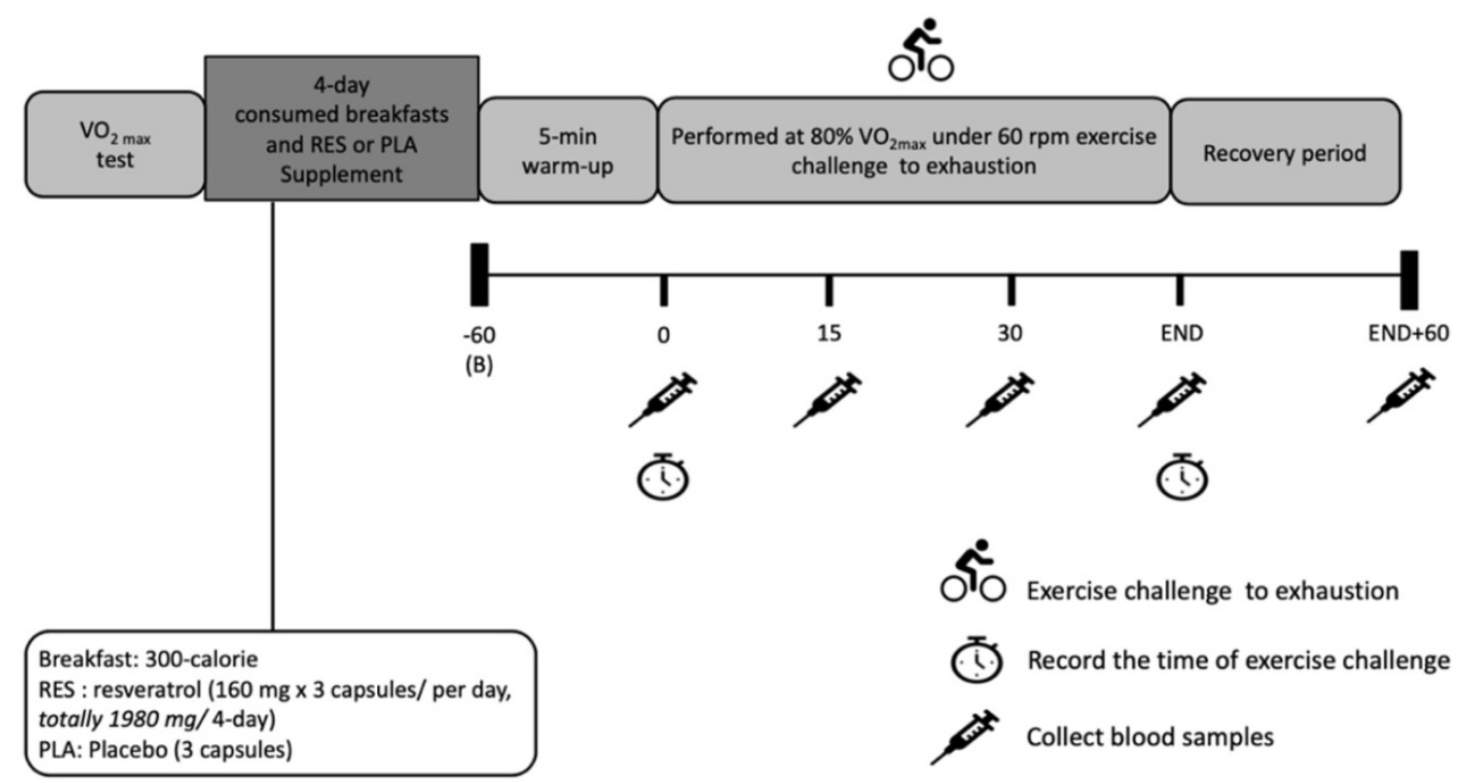

Figure 1. Experimental design and protocol. 
concentrations in the serum samples were calculated

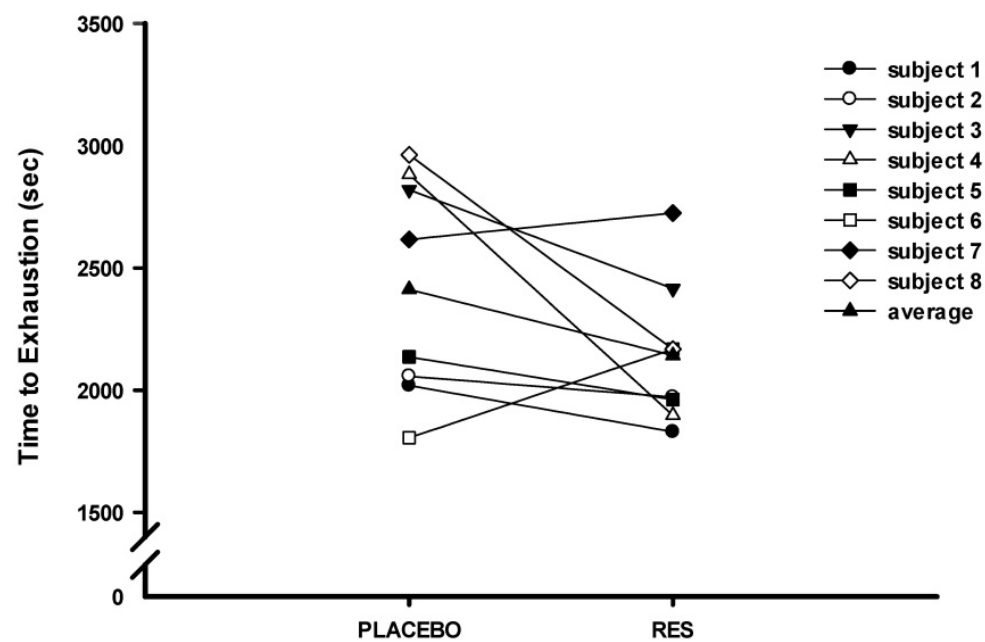

Figure 2. Individuals/average time to exhaustion (TTE) with $80 \% \dot{\mathrm{V}}_{2 \max }$ exercise challenge after placebo or resveratrol supplementation.
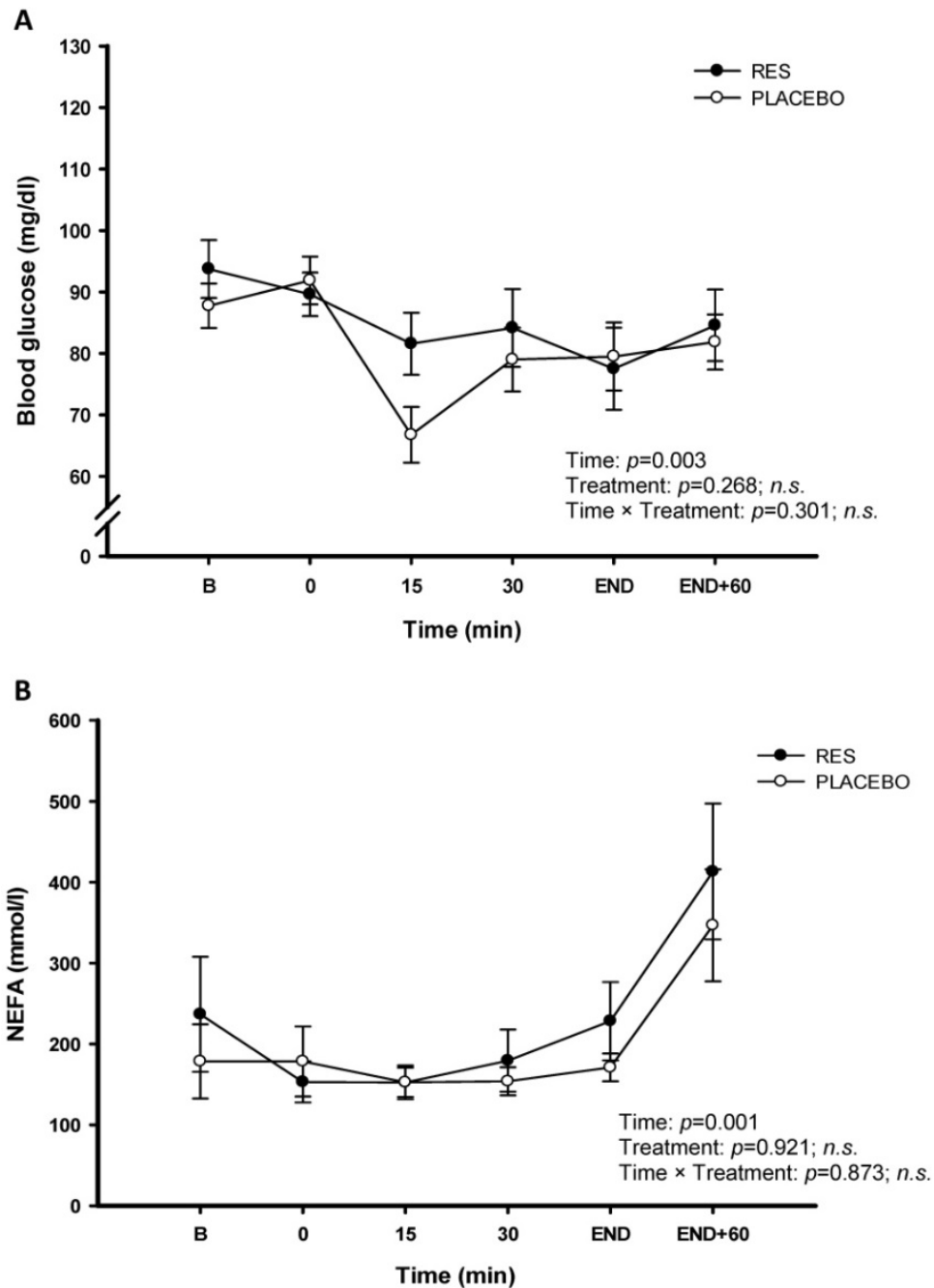

Figure 3. Blood glucose (A) and plasma non-esterified fatty acids (NEFA) (B) concentrations before and during exercise in resveratrol (-๑-) and placebo (-O-). B represents before exercise. END represents immediately finishing exercise. END +60 represents 60 minutes after exercise. Values are expressed as mean $\pm \mathrm{SE}, \mathrm{N}=8$. * Significant difference against placebo $(P<0.05)$. according to the standard curve. The concentrations of TNF- $\alpha$ and IL-6, which are cytokines, were measured using commercially available IL-6 (R \& D, HS 600C, Minneapolis, USA) and TNF-a (R \& D, HSTA00E, USA) reagents, respectively. An enzyme immunoassay (Tecan GENios, A-5082, Austria) was applied to measure the absorbances of IL-6 and TNF- $\alpha$ at 540 and $405 \mathrm{~nm}$, respectively; the concentrations of IL- 6 and TNF- $a$ in the serum samples were then calculated according to the standard curves.

\section{Statistical analysis}

All data were represented as mean with their standard errors (Mean \pm SE) using SPSS software (IBM SPSS Statistics; IBM Corp.). A paired $t$-test was conducted to examine the differences between the participants in the performance of cycling challenge. Two-way ANOVA with repeated measures was used to compare all blood samples. If a significant interaction was detected between the experimental trials and the time, a simple main effect analysis was conducted, and Fisher's post hoc test was used to distinguish significant difference between pairs of conditions. The a level was set at 0.05 to indicate a significant difference for all comparisons.

\section{Results}

\section{Effects of Resveratrol supplementation on cycling time to exhaustion and energy substrates metabolism}

The results of this study on the individuals' or statistically average exhausting time under a single bout of high-intensity cycling exercise challenge were not significantly increased after oral RES supplementation (RES: $2142.25 \pm 106.48$ (sec); Placebo: $2412.5 \pm 161.41$ (sec), $p>0.05$ ) (Figure 2). Identically, the blood glucose (Figure 3A) and NEFA (Figure 3B) did not significantly different response after oral RES supplementation compared to those of placebo treatment $(p>0.05)$.

\section{Resveratrol supplementation attenuates IL-6 but not oxidative stress}

The concentrations of the LDH (Figure 4A), CK (Figure 4B), and UA (Figure 4C) were no significant difference between RES and placebo $(p>0.05)$. The cycling exercise 
performance were not enhanced after 4-d RES supplementation, based on no difference in energy substrates metabolism and attenuating tendency in exercise induced fatigue between two trials. The changes in inflammation and oxidative stress were

$$
\text { A }
$$

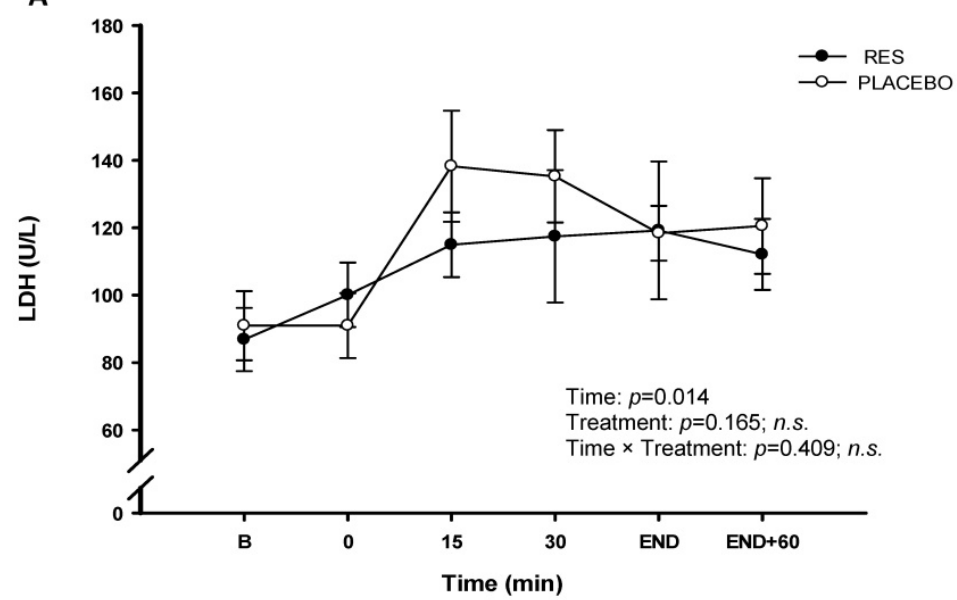

B

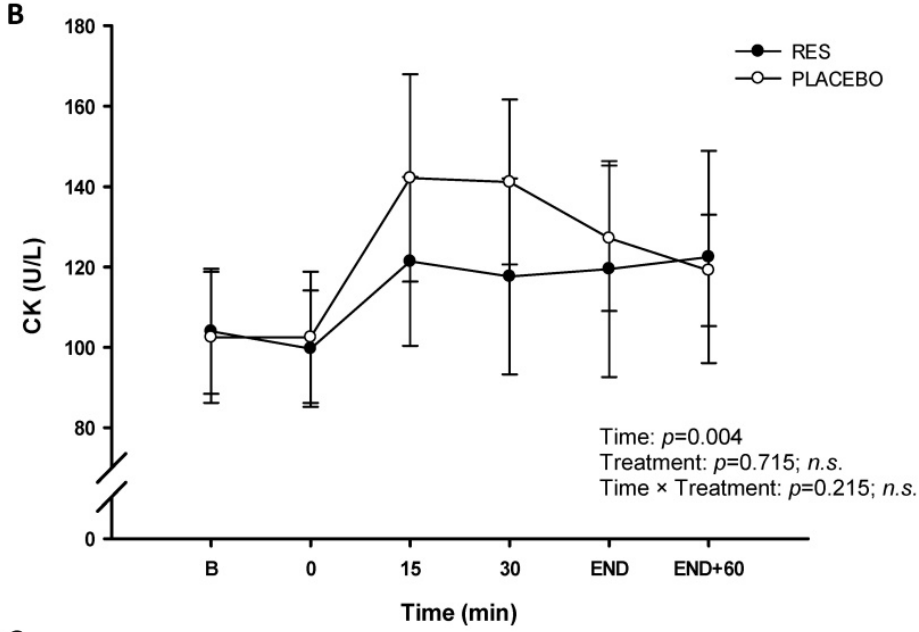

c

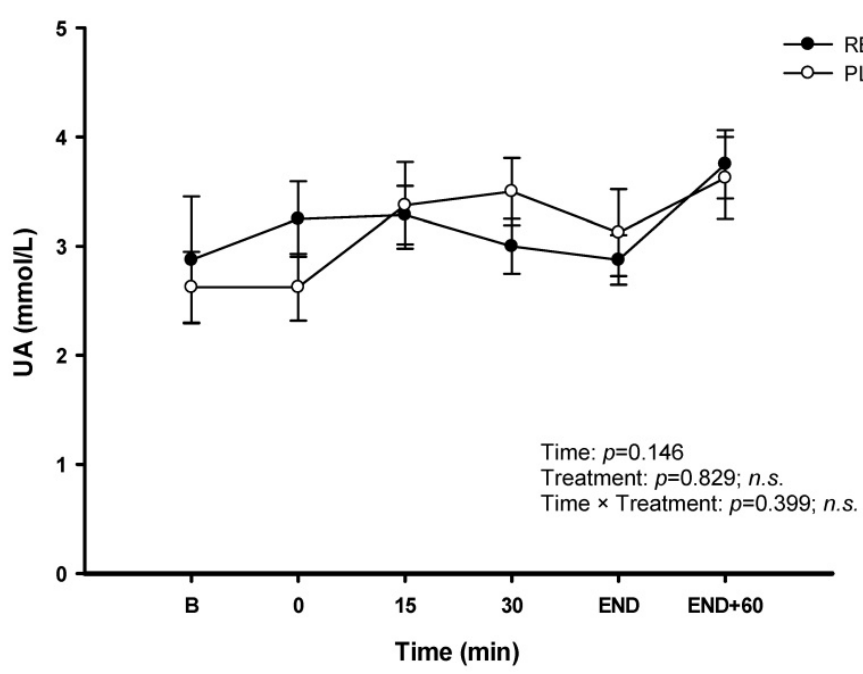

Figure 4. Serum lactate dehydrogenase (LDH) (A), creatine kinase (CK) (B) and uric acid (UA) (C) concentrations before and during exercise in resveratrol (-O-) and placebo (- $\bigcirc-)$. B represents before exercise. END represents immediately finishing exercise. END+60 represents 60 minutes after exercise. Values are expressed as mean $\pm \mathrm{SE}, \mathrm{N}=8$. * Significant difference against placebo $(P<0.05)$. measured during this high-intensity cycling exercise challenge. The figure 5 showed oxidative stress indicators TAC (Figure 5A) and MDA (Figure 5B). Exercise induced oxidative stress indicators TAC and MDA were shown that there were no significant differences between two trials $(p>0.05)$. Figure 6 showed no significant differences pro-inflammation indicators of TNF- $\alpha$ between two treatments (Figure 6A, $p>$ 0.05). However, there was significantly lower exercise-induced response in IL-6 after oral resveratrol supplementation (Figure 6B, $p<0.05$ ).

\section{Discussion}

In the present study, RES supplementation before the high-intensity exercise was assumed to enhance anti-oxidation and anti-inflammation capabilities, mitigate factors contributing to exercise-induced fatigue, and improve participants' cyclic exercise performance. After 4 days of receiving oral RES supplementation, the participants engaged in a high-intensity cycling challenge. The results were as follows: (1) the single bout of exercise performance with $80 \% \dot{\mathrm{VO}}_{2 \max }$ at 60 rpm did not be improved during the cycling challenge; (2) the blood parameters of glucose, NEFA, LDH, CK, and UA measured during exercise did not support the hypothesis that RES attenuates exercise fatigue; (3) the plasma TAC, MDA and TNF concentrations indicated that RES supplementation did not improve the antioxidant and anti-inflammation capabilities. (4) RES supplementation significantly decreased the IL-6 level induced by exercise stimulus. According to cell experiments, RES supplementation increased the number of muscle mitochondria in endothelial cells [21]. Low doses of RES supplementation significantly increased muscle endurance and power in swimming animals [10]. Cell and animal experiments have indicated that RES supplementation improves antioxidant [3] and anti-inflammation [22] capabilities in animals. In the present human experiment, RES supplementation did not effectively improve high-intensity cycling exercise performance or mitigate the exerciseinduced fatigue. However, RES supplementation seems to prevent muscle stress on lower IL-6 release, a cytokine with 
pro-inflammation, during the high-intensity cycling exercise challenge following 4-day oral RES supplementation.

IL-6 is a pro-inflammatory cytokine and muscle damage marker following exercise challenge [13], which has positive association with content of contracting skeletal muscle glycogen [23]. The rats study showed that a single bout of a 90-min downhill running eccentric exercise significantly increased mRNA IL-6 and TNF- $a$ but no change in serum levels of IL-6 and TNF-a [8]. The human study showed that $65 \%$ and $85 \%$ maximal power $\left(\mathrm{W}_{\max }\right)$ of knee extension exercise were performed for $35 \mathrm{~min}$ separately in seven healthy males and the authors inferred that IL-6 may be linked to the regulation of glucose homeostasis during exercise [24]. In our human study, the significantly lower IL-6 response during the exercise period was found after RES

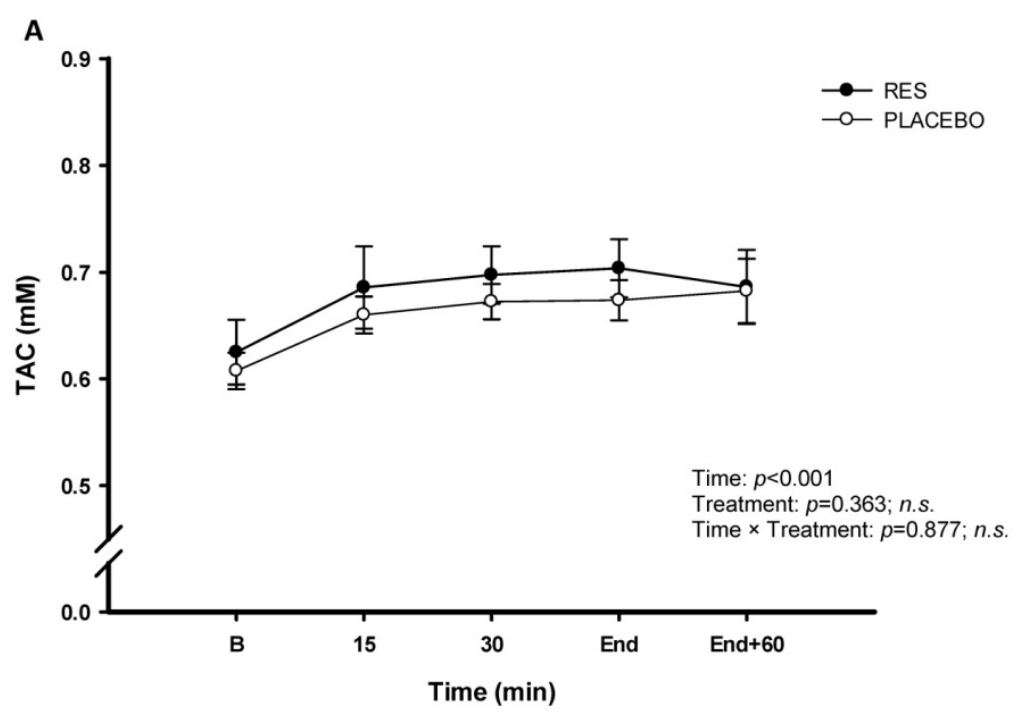

B

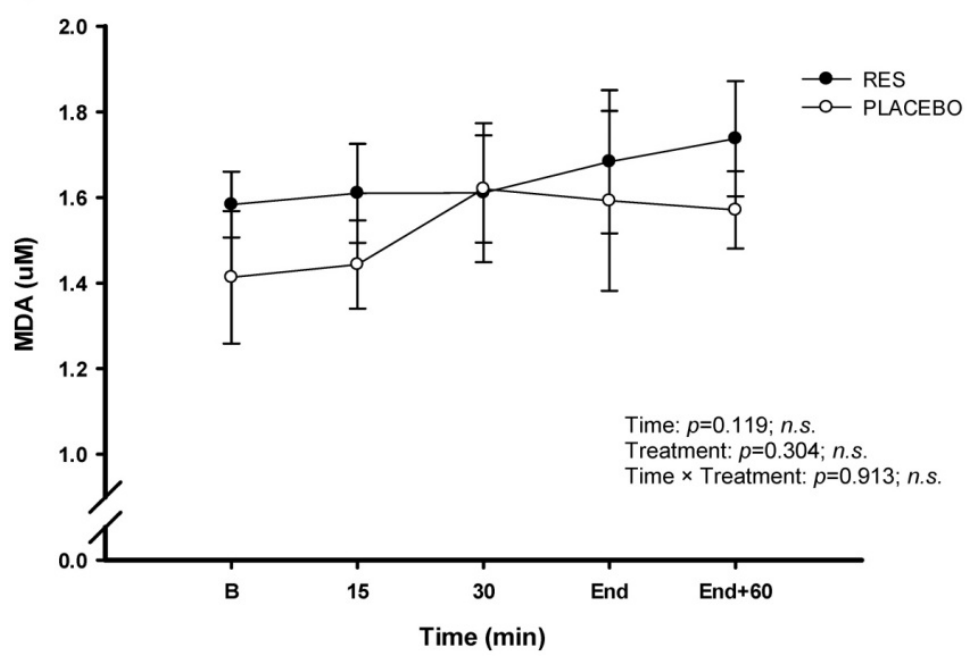

Figure 5. Serum total antioxidant capacity (TAC) (A) and malondialdehyde (MDA) (B) concentrations before and during exercise in resveratrol (-๑-) and placebo (-O-). B represents before exercise. END represents immediately finishing exercise. END +60 represents 60 minutes after exercise. Values are expressed as mean $\pm \mathrm{SE}, \mathrm{N}=8$. * Significant difference against placebo $(P<0.05)$. ingestion, we implied that 4-day RES supplementation prevented the release of cytokine with pro-inflammation during high-intensity cycling exercise. Although there was no measurement of muscle glycogen in the present study to support this positive effect of RES. However, IL-6 is an important metabolic role during exercise that mediates the effect of lipolysis, inflammation, and muscle glycogen metabolism [25]. Therefore, we infer that the significantly lower IL-6 during exercise in this study may be implied the 4-day RES supplementation have ergogenic property on preventing of muscle cytokine with proinflammation or attenuating on exercising muscle glycogen depletion during high intensity cycling exercise.

The individual observation of this study revealed that only two of the participants' have shown the improved cyclic exercise performance after the 4-day RES supplementation, and two of the remaining participants have very poor performance than those of placebo supplementation. This was possibly because the initial intensity of the bicycle ergometer overwhelmed the leg muscles of the participants, who expressed severe fatigue in their legs. Exercise fatigue is caused by the overconsumption of energy in human bodies during high-intensity exercise, which produces excessive free radicals and leads to high oxidation and inflammation in the body. During exercise, the glycogen in skeletal muscles is rapidly exhausted, causing rapid blood glucose consumption and accelerating lipolysis. Under this condition, NEFA concentration, which is a blood biochemical index for lipolysis, increases to provide the energy needed to maintain the exercise performance [26]. CK and LDH in the blood are a well-established marker of muscle damage used to infer damage to the sarcolemma. The significant changes of CK and LDH were observed when the load of a workout exceeding the body's capacity, which triggering the exercise-induced fatigue to attenuating on the high-intensity exercise performance [27]. High blood urea also indicates that more protein is broken down than is synthesized during exercise fatigue [28]. However, the blood glucose, NEFA, CK, $\mathrm{UA}$, and LDH concentrations measured in the present study could not confirmed the mitigating effect of RES supplementation 
on high-intensity exercise fatigue. There was no treatment effect of RES on the blood indices in the present human study. Although the application of nanoemulsifying-RES capsules eliminated problems related to RES absorbance and bioavailability $[9,10$, 29], RES supplementation did not mitigate the factors related to exercise fatigue or improve the exercise performance of the participants in the cycling challenge.

During high-intensity exercise periods, ammonia, a protein metabolite, is broken down into urea. Plasma levels of urea are known to increase in response to exercise. During fatiguing exercise, active skeletal muscle oxidation of purines increases, thus increasing the efflux of uric acid from skeletal muscle into the blood compartment [30], As uric acid is an antioxidant this should increase TAC [31]. In present human study, the data of TAC showed an increase over time during exercise period after RES
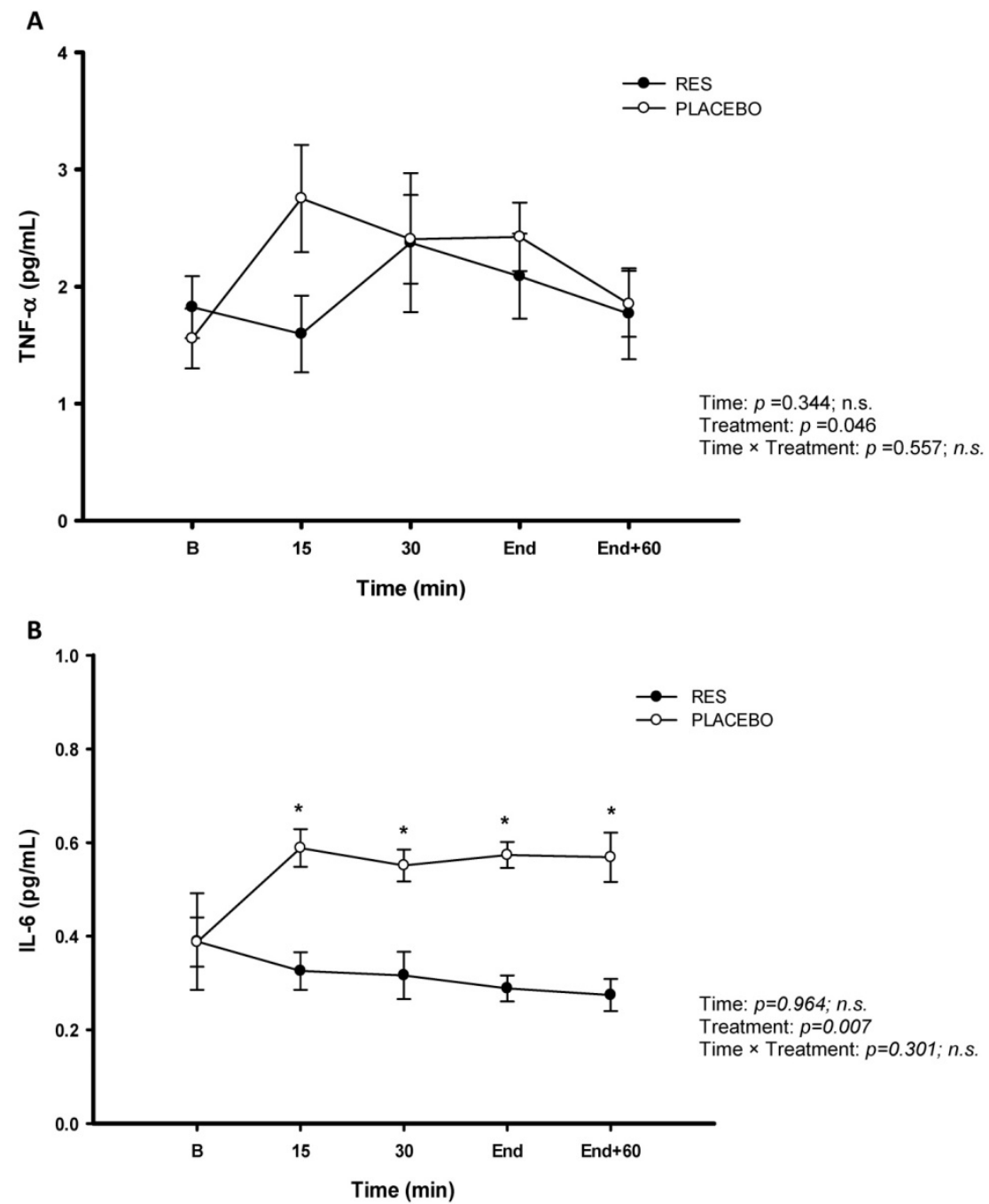

Figure 6. Serum tumor necrosis factor- $\alpha$ (TNF- $\alpha$ ) (A) and interleukin-6 (IL-6) (B) concentrations before and during exercise in resveratrol (- -) and placebo (- $\bigcirc-)$. B represents before exercise. END represents immediately finishing exercise. END +60 represents 60 minutes after exercise. Values are expressed as mean $\pm S E$, $\mathrm{N}=8$. * Significant difference against placebo $(P<0.05)$. supplementation (Figure 5A). Additionally, creatine kinase is a marker for a muscle tissue which may be correlated with physical training status, as depend on sarcomeric damage [32]. The highest post-exercise serum enzyme activities are found after prolonged exercise such as ultradistance marathon running or weight-bearing exercises $[33,34]$. LDH catalyzes the conversion of pyruvates to lactic acids and is an index of anaerobic metabolism during high-intensity exercise [35]. However, after 4-day RES supplementation, high $\mathrm{LDH}, \mathrm{UA}$, and CK concentrations were identified in the placebo groups during the cycling challenge. Although the LDH, UA, and CK concentrations in the RES group were not as high as those in the placebo group, no statistical difference was identified between the two groups. The results of our study implied the positive effect of 4-day RES supplementation could not be supported on attenuating exercise fatigue in exercising humans.

We speculated the dosage and supplement duration as the crucial factors in our human study from the previous resveratrol effect on human interventional trials [36]. The relevant human study revealed that 4 days RES (1920 mg totally) failed in enhancing muscle glycogen recovery and mitochondrial biosynthesis [12]. Therefore, the total dosage is insufficient on influencing the changes in exercising metabolites due to duration of the supplementation in the present study. Further human studies will be conducted using the recommended dosage of $60 \mathrm{mg} / \mathrm{d}$ for chronic effect and $2400 \mathrm{mg} / \mathrm{d}$ for acute effect of resveratrol [9, 37], which are warranted to confirm the ergogenic properties on endurance performance.

RES supplementation is likely to exert anti-fatigue pharmacological effect in animal studies $[9,10]$. In the present human study, we speculated that the same trend may occur in TAC, MDA, and TNF-a after RES supplementation. However, the 4-day RES supplementation in the present study did not show the positive effect under high-intensity cycling performing based on TAC, MDA and TNF-a. This was inconsistent with the findings of numerous cell and animal 
experiments, which have indicated that RES supplementation regulates antioxidant and anti-inflammatory functions [38, 39]. However, exercise-

induced IL-6 exists lower response in this study. Therefore, we conclude that 4-day oral RES supplementation can attenuate exercise-induced IL-6 response but not fatigue, oxidative stress. We inference that 4-day oral RES supplementation have positive influence on cytokine IL-6 but no ergogenic property on the high-intensity cycling exercise performance. However, this study also has limitations including the recruitment number of participates although a single-blind crossover study design was conducted. Further future studies need to attend to sample size. Similarly, there was a lack of a diet diary and records about physical activity for all participants. Based on the knowing variation of MDA as a measure of oxidative stress, diet and training diaries could provide detailed information that could clarify the shorter-term ergogenic property of resveratrol on oxygen stress, inflammation status and high-intensity cycling performance-enhancing in humans.

\section{Conclusions}

In summary, we demonstrate that 4-day oral RES supplementation (480 $\mathrm{mg} /$ day) is capable of attenuating the IL-6, cytokine with pro-inflammatory, during high-intensity cycling exercise. However, the given RES dosage and supplement duration appear to be insufficient to attenuate oxygen stress and fatigue during exercise. The final outcome demonstrated the 4-day oral RES supplementation ( $480 \mathrm{mg}$ / day) could serve as ergogenic aid on attenuating IL- 6 but not oxygen stress and fatigue during high-intensity cycling exercise. Since inflammation is an important factor for endurance performance, this finding could certainly beneficial to athletes, who are training for key competition.

\section{Abbreviations}

RES: resveratrol; NEFA: non-estrified fatty acid; LDH: lactate dehydrogenase; CK: creatine kinase; UA: uric acid; TAC: total antioxidant capacity; MAD: malondialdehyde; TNF- $\alpha$ : tumor necrosis factor- $\alpha$; IL-6: interleukin-6; RER: respiratory exchange ratio; RPE: rated perceived exertion.

\section{Acknowledgments}

This work was supported by the grants from the Ministry of Science and Technology (MOST 106-2410H-142-014-MY2 and MOST 109-2918-I-142-001) of Taiwan.

\section{Authorship}

All authors are qualified and approved this final submitted version of this study. Chia-Chen Liu, Chun-Ching Huang and I-Shiung Cheng designed the experiments. Jung-Piao Tsao and Chia-Chen Liu carried out the laboratory experiments. Jung-Piao Tsao, and Chun-Ching Huang contributed reagents, materials and analysis platforms. Jung-Piao Tsao, Chia-Chen Liu and Chun-Ching Huang analyzed the data. Jeffrey R. Bernard helped with the polishing of English writing. I-Shiung Cheng interpreted the results, prepared the figures, wrote and revised the manuscript.

\section{Competing Interests}

The authors have declared that no competing interest exists.

\section{References}

1. Bavaresco L. Role of viticultural factors on stilbene concentrations of grapes and wine. Drugs Exp. Clin. Res. 2003; 29(5-6): 181-187.

2. De La Lastra CA, Villegas I. Resveratrol as an antioxidant and pro-oxidant agent: mechanisms and clinical implications. Biochem Soc Trans. 2007; 35(5): 1156-1160.

3. Das S, Das DK. Anti-inflammatory responses of resveratrol. Inflamm Allergy Drug Targets. 2007; 6: 168-173.

4. Brasnyó P, Molnár GA, Mohás M, Markó L, Laczy B, Cseh J, et al. Resveratrol improves insulin sensitivity, reduces oxidative stress and activates the Akt pathway in type 2 diabetic patients. Br. J. Nutr. 2011;106(3): 383-389.

5. Timmers S, Konings E, Bilet L, Houtkooper RH, van de Weijer T, Goossens $\mathrm{GH}$, et al. Calorie restriction-like effects of 30 days of resveratrol supplementation on energy metabolism and metabolic profile in obese humans. Cell Metab. 2011; 14(5): 612-22.

6. Vitaglione P, Sforza S, Galaverna G, Ghidini C, Caporaso N, Vescovi PP, et al. Bioavailability of trans-resveratrol from red wine in humans. Mol. Nutr. Food Res. 2005; 49(5): 495-504.

7. Powers SK, Nelson WB, Hudson MB. Exercise-induced oxidative stress in humans: cause and consequences. Free Radic Biol Med. 2011; 51(5): 942-950.

8. Zuo Q, Qu F, Li N, Wang S, Liu J, Xu C, et al. Eccentric exercise results in a prolonged increase in interleukin- 6 and tumor necrosis factor-a levels in rat skeletal muscle. J Muscle Res Cell Motil. 2019; 40(3-4): 379-387.

9. Yen CC, Chang CW, Hsu MC, Wu YT. Self-nanoemulsifying drug delivery system for resveratrol: enhanced oral bioavailability and reduced physical fatigue in rats. Int J Mol Sci. 2017; 18(9): 1853.

10. Wu RE, Huang WC, Liao CC, Chang YK, Kan NW, Huang CC. Resveratrol protects against physical fatigue and improves exercise performance in mice. Molecules. 2013;18(4):4689-4702.

11. Scribbans TD, Ma JK, Edgett BA, Vorobej KA, Mitchell AS, Zelt JG, et al. Resveratrol supplementation does not augment performance adaptations or fibre-type-specific responses to high-intensity interval training in humans. Appl Physiol Nutr Metab. 2014; 39(11): 1305-1313.

12. Huang CC, Liu CC, Tsao JP, Hsu CL, Cheng IS. Effects of Oral Resveratrol Supplementation on Glycogen Replenishment and Mitochondria Biogenesis in Exercised Human Skeletal Muscle. Nutrients.2020; 12(12):3721.

13. Cipryan L. IL-6, antioxidant capacity and muscle damage markers following high-intensity interval training protocols. J Hum Kinet. 2017; 56: 139-48.

14. Steensberg A, Febbario M, van Hall G, Osada T, Sacchetti M, Saltin B, Pedersen BK. Interleukin- 6 production in contracting human skeletal muscle is influenced by pre-exercise muscle glycogen content. J. Physiol. (London) .2001; 537: 633-639.

15. Brandt C, Pedersen BK. The role of exercise-induced myokines in muscle homeostasis and the defense against chronic diseases. J Biomed Biotechnol 2010; 2010: 520258

16. Pedersen BK, Akerstrom TC, Nielsen AR, Fischer CP. Role of myokines in exercise and metabolism. J Appl Physiol. 2007; 103(3): 1093-1090.

17. Kitada M, Kume S, Imaizumi N, Koya D. Resveratrol improves oxidative stress and protects against diabetic nephropathy through normalization of Mn-SOD dysfunction in AMPK/SIRT1-independent pathway. Diabetes. 2011; 60(2): 634-643.

18. Dal-Ros S, Zoll J, Lang AL, Auger C, Keller N, Bronner C, Geny B, Schini-Kerth VB. Chronic intake of red wine polyphenols by young rats prevents aging-induced endothelial dysfunction and decline in physical performance: Role of NADPH oxidase. Biochem Biophys Res Commun. 2011; 404: 743-749. 
19. Kan NW, Ho CS, Chiu YS, Huang WC, Chen PY, Tung YT, Huang CC. Effects of Resveratrol Supplementation and Exercise Training on the Exercise Performance in Middle-aged Mice. Molecules.2016; 21(5): 661.

20. Zuniga JM, Housh TJ, Camic CL, Bergstrom HC, Traylor DA, Schmidt RJ, et al. Metabolic parameters for ramp versus step incremental cycle ergometer tests. Appl Physiol Nutr Metab. 2012; 37(6): 1110-1117.

21. Csiszar A, Labinskyy N, Pinto JT, Ballabh P, Zhang H, Losonczy G, et al. Resveratrol induces mitochondrial biogenesis in endothelial cells. Am J Physiol Heart Circ Physiol. 2009; 297(1): H13-H20.

22. Das S, Das DK. Anti-inflammatory responses of resveratrol. Inflamm Allergy Drug Targets. 2007; 6(3): 168-173.

23. Keller C, Steensberg A, Pilegaard H, Osada T, Saltin B, Pedersen BK, Neufer PD. Transcriptional activation of the IL-6 gene in human contracting skeletal muscle: influence of muscle glycogen content. FASEB J. 2001; 15: 2748-2750.

24. Helge JW, Stallknecht B, Pedersen BK, Galbo H, Kiens B and Richter EA. The effect of graded exercise on IL-6 release and glucose uptake in skeletal muscle. J Physiol. 2003; 546(1): 299-305.

25. Pedersen BK, Steensberg A, Fischer C, Keller C, Keller P, Plomgaard P, Wolsk-Petersen E, Febbraio M. The metabolic role of IL-6 produced during exercise: is IL-6 an exercise factor? Proc Nutr Soc. 2004; 63(2): 263-267.

26. Pösö AR, Hyyppä S, Geor RJ. Metabolic responses to exercise and training. Saunders, Philadelphia. 2004:248-273.

27. Del Coso J, Fernandez de Velasco D, Abian-Vicen J, Salinero JJ, Gonzalez-Millan C, Areces F, et al. Running pace decrease during a marathon is positively related to blood markers of muscle damage. PloS one. 2013; 8(2): e57602.

28. Haralambie G, Berg A. Serum urea and amino nitrogen changes with exercise duration. Eur. J. Appl Physiol. 1976; 36(1): 39-48.

29. Summerlin N, Soo E, Thakur S, Qu Z, Jambhrunkar S, Popat A. Resveratrol nanoformulations: challenges and opportunities. Int J Pharm. 2015; 479(2): 282-290.

30. Duthie GG, Robertson JD, Maughan RJ, Morrice PC. Blood antioxidant status and erythrocyte lipid peroxidation following distance running. Arch Biochem Biophys. 1990; 82: 78-83.

31. Cao G, Prior RL. Comparison of the analytical methods for assessing the total antioxidant capacity of human serum. Clin. Chem. 1998; 44: 1309-1315.

32. Vincent HK, Vincent KR. The effect of training status on the serum creatine kinase response, soreness and muscle function following resistance exercise. Int J Sports Med.1997; 18: 431-437.

33. Magrini D, Khodaee M, San-Millan I, Hew-Butler T, Provance AJ. Serum creatine kinase elevations in ultramarathon runners at high altitude. Phys Sportsmed. 2017; 45(2):129-133.

34. Callegari GA, Novaes JS, Neto GR, Dias I, Garrido ND, Dani C. Creatine Kinase and Lactate Dehydrogenase Responses After Different Resistance and Aerobic Exercise Protocols. J. Hum. Kinet. 2017; 58: 65-72.

35. Stanley WC. Myocardial lactate metabolism during exercise. Med Sci Sports Exerc. 1991; 23(8): 920-924

36. Ramírez-Garza SL, Laveriano-Santos EP, Marhuenda-Muñoz M, Storniolo CE Tresserra-Rimbau A, Vallverdú-Queralt A, Lamuela-Raventós RM. Health Effects of Resveratrol: Results from Human Intervention Trials. Nutrients. 2018; 10: 1892.

37. Bicer M, Baltaci SB, Mogulkoc R, Baltaci AK, Avunduk MC. Effect of resveratrol administration on muscle glycogen levels in rats subjected to acute swimming exercise. Cell. Mol. Biol. 2019; 65: 28-31.

38. Soufi FG, Vardyani M, Sheervalilou R, Mohammadi M, Somi MH. Long-term treatment with resveratrol attenuates oxidative stress pro-inflammatory mediators and apoptosis in streptozotocin-nicotinamide-induced diabetic rats. Gen Physiol Biophys. 2012; 31(4): 431-438.

39. Tomé-Carneiro J, Larrosa M, Yáñez-Gascón MJ, Dávalos A, Gil-Zamorano I, Gonzálvez M, et al. One-year supplementation with a grape extract containing resveratrol modulates inflammatory-related microRNAs and cytokines expression in peripheral blood mononuclear cells of type 2 diabetes and hypertensive patients with coronary artery disease. Pharmacol Res. 2013; 72: 69-82. 